\title{
HSV Pseudotumor of the Hypopharynx: A Diagnostic Challenge
}

\author{
Mark Knackstedt ${ }^{1}$, Syed Naqvi ${ }^{1}$, and Ron Karni ${ }^{1}$ \\ ${ }^{1}$ University of Texas McGovern Medical School
}

November 17, 2020

\begin{abstract}
A 50-year-old male presented with HIV and an ulcerative mass on the hypopharynx. Initially thought to be malignant, multiple biopsies and surgeries over 19 months did not resolve the lesion. Discovery of the cytopathic effect on biopsy led to diagnosis of HSV pseudotumor. The lesion resolved with valacyclovir treatment.

HSV Pseudotumor of the Hypopharynx: A Diagnostic Challenge

Short running title: HSV Pseudotumor of the Hypopharynx

Mark I Knackstedt BS ${ }^{1}$, Syed HS Naqvi MD${ }^{1}$, Ron J Karni MD ${ }^{1}$

${ }^{1}$ Department of Otorhinolaryngology - Head \& Neck Surgery, McGovern Medical School at the University of Texas Health Science Center Houston, Houston, Texas 77030

Corresponding Author:

Department of Otorhinolaryngology - Head and Neck Surgery - McGovern Medical School 6400 Fannin St. St. 2700, Houston, TX 77030 Syed.Naqvi@uth.tmc.edu (281) 925-8309
\end{abstract}

Keywords: herpes simplex virus; HIV; pharynx; pseudotumor; acyclovir

Key Clinical Message: HSV in the context of an immunosuppressed patient can cause an inflammatory pseudotumor. These pseudotumors are rare inflammatory reactions and can be mistaken for malignancy. Addressing the cause of inflammation will resolve the lesion.

Please refer all correspondence, reprint requests, and proofs to Syed Naqvi at the Department of Otorhinolaryngology - Head and Neck Surgery, McGovern Medical School, 6400 Fannin St., Ste. 2700, Houston, TX 77030 - email: Syed.Naqvi@uth.tmc.edu - phone - (281) 925-8309

Abstract:

A 50-year-old male presented with HIV and an ulcerative mass on the hypopharynx. Initially thought to be malignant, multiple biopsies and surgeries over 19 months did not resolve the lesion. Discovery of the cytopathic effect on biopsy led to diagnosis of an HSV pseudotumor. The lesion resolved with valacyclovir treatment.

Introduction:

The high rate of herpes simplex virus (HSV) seropositivity in the United States makes it likely most surgeons and clinicians will deal with this disease and its sequelae. The most common sequelae of HSV infection of the oral cavity and pharynx is pharyngitis with vesicles and large, painful, shallow ulcers. ${ }^{1}$ However, in cases of immunosuppression, atypical presentations of HSV are common and new sequelae are still being discovered. Patients with HSV are more likely to transmit human immunodeficiency virus (HIV), and the 
two viruses are frequently seen in conjunction. Recurrent ulcerative HSV is even recognized as a possible early sign for immunosuppression. In these coinfected individuals, rare hypertrophic pseudotumor forms have been described in the genital region and oral cavity. ${ }^{1-4}$ These rare diagnoses are often undiagnosed and unaddressed in this population. We report a case of detrimental hypopharyngeal pseudotumor development secondary to and HSV infection in the presence of long-term HIV infection.

Case Report:

A 50-year-old African American male with a history of HIV, HSV, seizures and smoking presented to the emergency room July 2020 for a 3-week history of progressive dysphagia, nausea, and vomiting. His past medical history was significant for a HAART controlled HIV infection diagnosed in 2004, a partial penectomy due to a large necrotic HSV lesion which had been recurring since 2000 and an ulcerative right hypopharyngeal mass found in February 2019. In February 2019, he was found face down beside his car with dyspnea and blood coming from the mouth. Rigid laryngoscopy revealed an ulcer with heaped edges and granulation tissue extending from the posterior pharyngeal wall down from the level of the tongue base to the left hypopharynx and pyriform sinus. He was given a tracheostomy and a biopsy of the ulcer was taken. Subsequent exploration of the central neck for hypopharyngeal injury did not yield any results. Pathology showed eosinophils and squamous epithelium on a background of plasma cells without signs of malignancy. He passed a swallow study then was decannulated and discharged to a rehabilitation center.

He was lost to follow up until July 2020 when he presented with progressive dysphagia. A CT of the head and neck with contrast revealed an extensive heterogeneously attenuating ulcerative mass filling the supraglottic larynx with transglottic extension into the pyriform sinus and hypopharynx. He was given a PEG and tracheostomy. A laryngoscopy at the time revealed a large ulcerative mass on the right hypopharynx with edema of the epiglottis and aryepiglottic folds. Several biopsies were taken, and pathology found lymphocytes positive for CD45 and variably positive for CD3 and CD20, but was negative for malignancy. He did not follow up again until September 2020 where a foul smell was noted. (Figure 1) At this time a neck CT with contrast revealed the ulcerated mass had expanded to the point it was completely transglottic with central nonnecrotic lymphadenopathy. A CD4+ count at the time was 259 and his virus load was undetectable. He returned to the OR for another biopsy. Pathology found squamous mucosa with ulceration, granulation tissue and extensive necrosis with sheets of eosinophils and plasma cells. It was found the herpes virus cytopathic effect was present and diagnosis was confirmed with immunohistochemical stain. The patient was diagnosed with pseudotumor of the hypopharynx secondary to HSV. He was then provided valacyclovir 1,000 mg q12 to help control the ulcerative mass.

On follow up 4 weeks later, the mass had shrunken in size. This allowed for increased visualization of the epiglottis. (Figures 2 and 3 ) The patient reported decreased dyspnea and cough with increased energy.

\section{Discussion}

Though atypical presentations of HSV infections in the setting of concomitant HIV infection have been described, no HSV induced pseudotumors of the pharynx have been described in the literature. This makes diagnosis of this case difficult as there are several other causes of neck masses seen in patients infected with HIV.

In the setting of an HIV infected patient, pharyngeal masses can often be caused by non-Hodgkin lymphoma, Kaposi sarcoma, p16 positive squamous cell carcinoma or mycobacterium tuberculosis infection. ${ }^{5}$ Biopsy for pharyngeal masses in HIV positive patients is prudent and required as a primary diagnostic step. If negative, the next best step is to consider possible opportunistic infections.

Husak et al. were the first to describe an exophytic mass caused by HSV acting as an opportunistic infection in an HIV positive patient. The patient was obtaining treatment for genital complications of HSV when the mass was noticed on his oral tongue. Pathology revealed eosinophilic granulocytes, lymphocytes, and plasma cells without signs of squamous cell carcinoma. He was given $300 \mathrm{mg}$ brivudin daily to resolve the infection. $^{2}$ This has several characteristics similar to our case and highlights the importance of the history 
of possible opportunistic infections in HIV positive patients with atypical presentations.

Tabaee et al. reported a case of a large exophytic mass formation of the oral tongue status post cardiac transplant with immunosuppression. Pathology was negative for carcinoma, but the squamous cells displayed changes indicative of HSV infection. The patient was given $350 \mathrm{mg}$ acyclovir IV BID and then $400 \mathrm{mg}$ of acyclovir po bid. The mass resolved completely. ${ }^{1}$ This highlights the need to consider HSV as an opportunistic infection in cases of immunosuppression outside HIV.

HSV infection of the genitalia causing large fungating masses are more common than oral or pharyngeal HSV masses. History of genital mass formation secondary to HSV may be an early sign of disseminated HSV infection causing further complications. ${ }^{3}$ In the case presented, the patient had an HSV-caused mass requiring removal several years before presentation of the airway mass.

Conclusion

The formation of pseudotumors in the presence of concomitant HSV infection and immunosuppression is a rare phenomenon with potentially severe effects. Although this process has been described in the genital region and, to a lesser extent, the oral cavity, it has not yet been described in the pharynx. We recommend testing and treatment for HSV infection in biopsy negative immunosuppressed patients with an unexplained mass with valacyclovir.

\section{Declarations}

Ethics approval and consent to participate - This study was conducted with the approval of the approval of the UT Health IRB (ID: IRB00000308)

Consent for publication - Not applicable

Competing interests - The authors declare that they have no competing interests or conflicts of interest.

Funding - There was no funding involved in this work.

Authors' contributions - MK: writing - original draft, methodology, formal analysis, resources, writing - review and editing, image processing; SN: conceptualization, methodology, writing - review and editing; RK: conceptualization, methodology, validation, visualization, project administration, writing - review and editing.

Acknowledgements: None

References

1. Tabaee A, Saltman B, Shutter J, Hibshoosh H, Markowitz A. Recurrent oral herpes simplex virus infection presenting as a tongue mass. Oral Surg Oral Med Oral Pathol Oral Radiol Endod. 2004 Mar;97(3):376-80. doi: 10.1016/j.tripleo.2003.11.007. PMID: 15024363.

2. Husak R, Tebbe B, Goerdt S, Wölfer LU, Zeichardt H, Stöffler-Meilicke M, Orfanos CE. Pseudotumour of the tongue caused by herpes simplex virus type 2 in an HIV-1 infected immunosuppressed patient. Br J Dermatol. 1998 Jul;139(1):118-21. doi: 10.1046/j.1365-2133.1998.02327.x. PMID: 9764162.

3. Barroso Dos Reis HL, Tosato Boldrini NA, da Silva Campos LC, Campos LG, Salume Costa FL, Jacomele Caldas JV, Pedras Almeida SL, Miranda AE. Hypertrophic genital herpes in an HIV-infected female patient: Imiquimod as an alternative treatment. Int J Infect Dis. 2020 Jun;95:153-156. doi: 10.1016/j.ijid.2020.04.025. Epub 2020 Apr 18. PMID: 32311452.

4. Sbidian E, Battistella M, Legoff J, Lafaurie M, Bézier M, Agbalika F, Simon F, Bouscarat F, Cayuela JM, Carcelain G, Houhou N, Bagot M, Molina JM, Janier M, Bachelez H. Recalcitrant pseudotumoral anogenital herpes simplex virus type 2 in HIV-infected patients: evidence for predominant B-lymphoplasmocytic infiltration and immunomodulators as effective therapeutic strategy. Clin Infect Dis. 2013 Dec;57(11):1648-55. doi: 10.1093/cid/cit592. Epub 2013 Sep 24. PMID: 24065320. 
5. Purgina B, Pantanowitz L, Seethala RR. A Review of Carcinomas Arising in the Head and Neck Region in HIV-Positive Patients. Patholog Res Int. 2011;2011:469150. doi: 10.4061/2011/469150. Epub 2011 May 10. PMID: 21660273; PMCID: PMC3108450.

\section{Figures}

Figure 1. The HSV induced pseudo tumor (left) obstructing view of the epiglottis in front of the base of tongue (bottom) taken in September 2020.

Figure 2. Shrinkage of the mass allowing for superior visualization of the epiglottis and hypopharynx after 4 weeks of acyclovir therapy in October 2020.

Figure 3. A small residual ulcerated mass (top) was still visible after treatment.

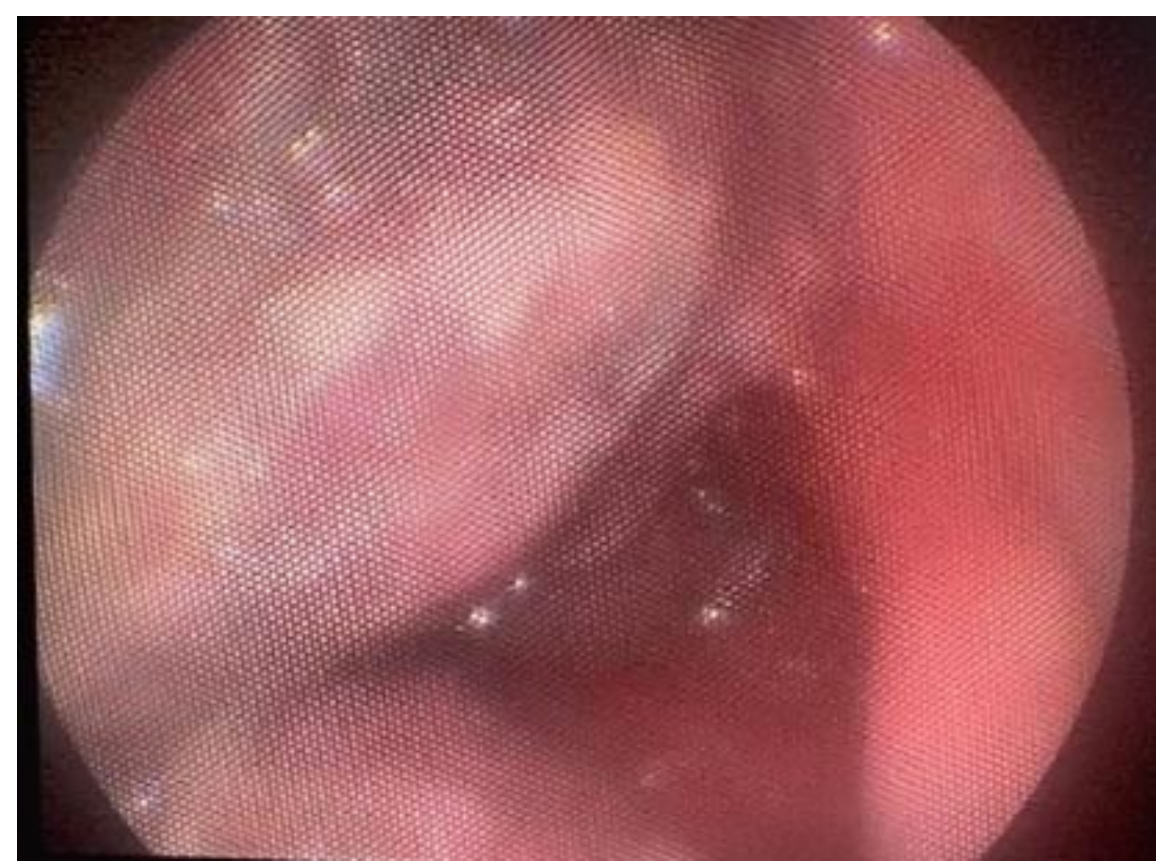




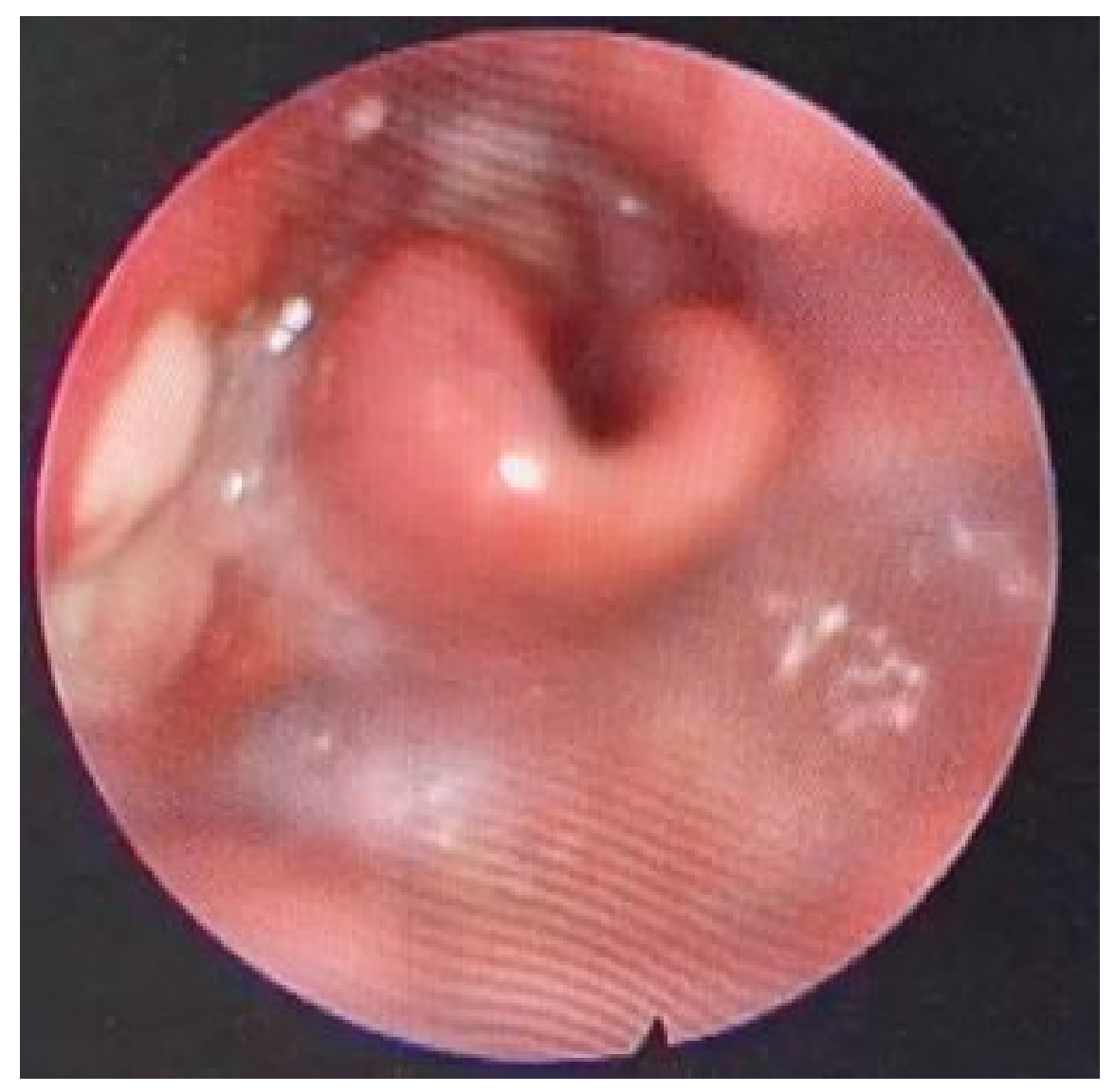




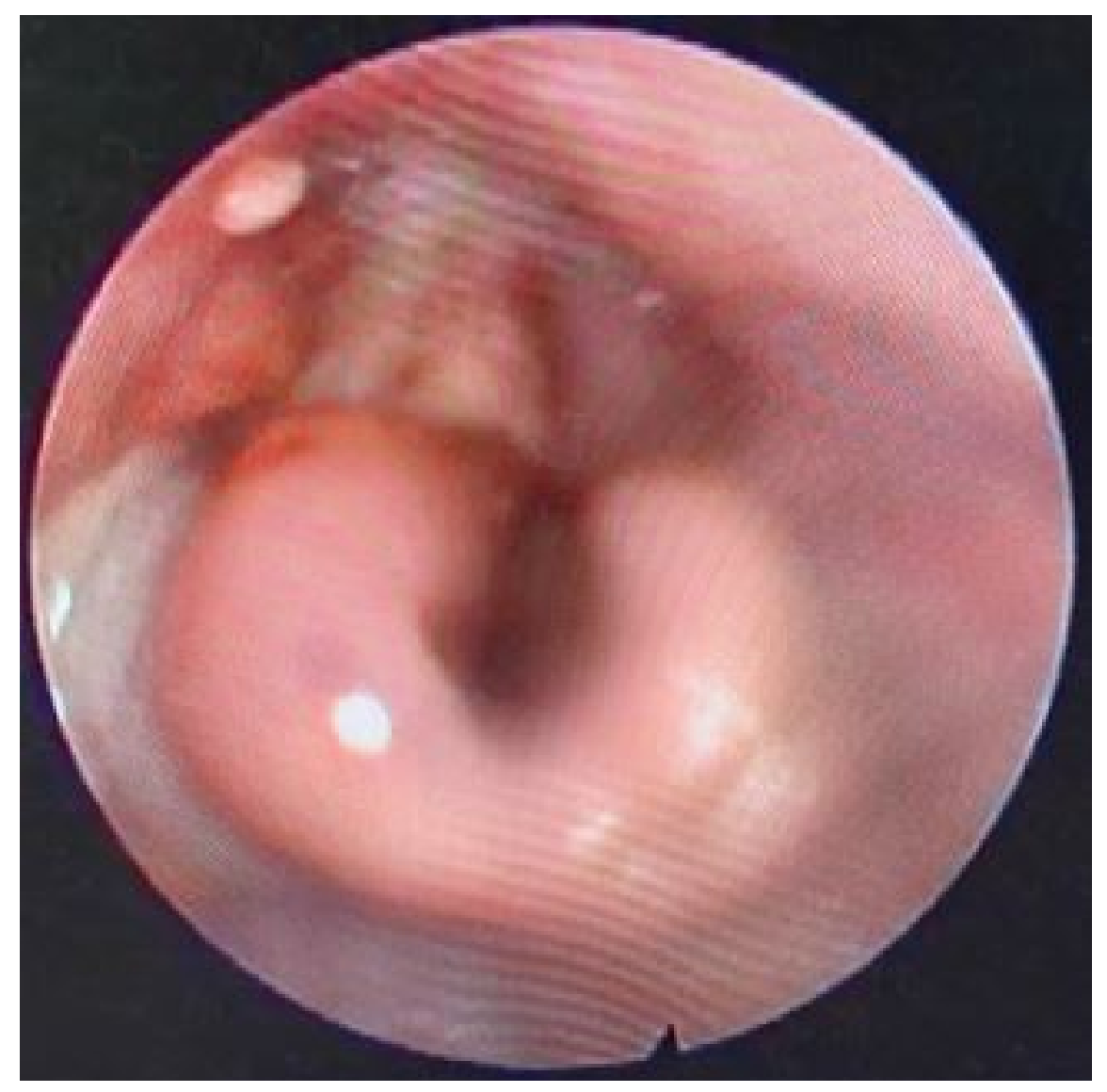

